\title{
LOS DIARIOS DE ALFOXDEN Y GRASMERE: ¿EJEM- PLOS DE LA POSICIÓN EPISTEMOLÓGICA PRIVILE- GIADA DE DOROTHY WORDSWORTH FRENTE AL ENTORNO NATURAL?
}

\author{
Margarita Carretero González, Universidad de Granada ${ }^{7}$ \\ Email: carreter@ugr.es
}

\begin{abstract}
Resumen: Este artículo explora la posibilidad de leer los diarios que Dorothy Wordsworth escribió en Alfoxden y Grasmere entre 1798 y 1803 como ejemplos de la posición epistemológica privilegiada que algunos postulados ecofeministas suponen a las mujeres, al compartir estas con la naturaleza no-humana la condición de sujetos oprimidos por el patriarcado. Si bien este postulado puede considerarse reduccionista si se acepta sin matices, si tenemos en cuenta los motivos que llevaron a Dorothy a escribir los diarios así como el papel que desempeñó en la evolución experimentada por el propio William en su forma de mirar el entorno natural, la lectura de los diarios desde esta perspectiva puede dar frutos interesantes. Ciertamente, la escritura de Dorothy estuvo siempre al servicio de la de su hermano pero, tal y como William admitió, la forma en la que su hermana se relacionaba con la naturaleza fue decisiva en el despertar de su propia sensibilidad.

Palabras clave: Dorothy Wordsworth, ecocrítica, ecofeminismo, diario, escritura de la naturaleza.
\end{abstract}

Título en inglés: “The Alfoxden and Grasmere Journals: examples of Dorothy Wordsworth's privileged epistemological perspective on the natural world?"

Abstract: This article explores the possibility of reading Dorothy Wordsworth's Alfoxden and Grasmere journals, written between 1798 and 1803, as instances of the privileged epistemological position that some ecofeminists ascribe to women, given the condition of oppressed subjects within patriarchy they share with non-human nature. Even if this premise may appear reductionist if assumed without nuances, the particular reasons that moved Dorothy to write her journals and the decisive role her brother William admitted she played in his evolution as a poet renders a reading of the journals from this standpoint particularly fruitful. Dorothy put her own work entirely at her brother's service, and William himself admitted to the role her relationship with the environment played in his own awakening to the natural world.

Keywords: Dorothy Wordsworth, ecocriticism, ecofeminism, journal, nature writing.

Date of reception: 16 July 2014

Date of acceptance: 8 September 2014 
En el capítulo quinto de Feminism and Ecology (1997), Mary Mellor plantea la cuestión que ha llevado a muchas ecofeministas a entrar en un terreno contencioso dentro de las teorías feministas: la defensa de que las mujeres ocupan una posición epistemológica privilegiada en relación con el mundo natural que las transforma en sus mejores defensoras puesto que son víctimas de la misma opresión patriarcal. La diferencia principal entre las distintas vertientes del ecofeminismo radica en si la asociación mujer-naturaleza es o no reivindicada, dependiendo de si los postulados beben o no de previas teorías feministas de la diferencia. Así pues, frente a algunas voces representativas de los ecofeminismos esencialistas surgidos del feminismo más radical de los años setenta (cf. Daly 1978, Griffin 1978, Starhawk 1979) que reclaman e invitan a celebrar esta asociación, las teorías que Alicia Puleo (2000: 130) ha llamado "de integración crítica" expuestas, entre otras por Ynestra King, Val Plumwood o Karen Warren advierten sobre lo peligroso de esta asociación "puesto que contribuye a perpetuar ese segundo plano en el que se encuentran las mujeres" (Carretero González 2010: 181-182). Mellor añade que lo esencialista de la asociación niega incluso la diferencia entre las distintas experiencias de mujeres, al tiempo que resulta reduccionista al dar prioridad al punto de vista de la mujer frente al de cualquier otro grupo que comparta esa misma sensación de opresión a la que la naturaleza no-humana está sujeta (Mellor 1997: 102-103). Partiendo de la base de que cualquier posición - privilegiada o no - frente a un fenómeno determinado responde a multitud de causas, este postulado ecofeminista se presenta como un interesante punto de partida para explorar la posición de Dorothy Wordsworth (1771-1855) ante al entorno natural de que dejó constancia en los diarios escritos en Alfoxden y Grasmere entre 1798 y 1803. Dada su particular posición social - privilegiada con respecto a otras mujeres de su misma clase social pero últimamente subordinada a la figura masculina de su hermano - y teniendo en cuenta que el propio William reconoció en los famosos versos de The Prelude el papel crucial que su hermana desempeñó en su evolución como poeta cuando asegura. "She, in the midst of all, preserved me still / A Poet, made me seek beneath that name / My office upon earth, and nowhere else" (Libro 10, vv. 919-921), y en "The Sparrow's Nest" recuerda que fue Dorothy quien le enseñó a mirar, a escuchar y, por consiguiente, a apreciar el entorno natural, es posible buscar en los diarios esa posición epistemológica de la que William bebería para luego trasladarla a sus propios poemas.

En tiempos recientes, la tendencia de la crítica a la hora de evaluar la producción de ambos Wordsworth ha sido la de subrayar la influencia mutua en lugar de seguir relegando a Dorothy a la posición secundaria que tradicionalmente había adoptado. Tal y como ya sugiriera Virginia Woolf, no compartieron el mismo tipo de discurso, pero sí el mismo temperamento artístico: Dorothy "stored [it] in prose and later William came and bathed in it and made it into poetry" (Woolf 1986: 169). Ciertamente, Dorothy no sólo escribió para poner sus observaciones al servicio de su hermano, un servicio que también acabó prestando a Coleridge puesto que ambos utilizaron las frases y descripciones que Dorothy había escrito en sus diarios para sus propias composiciones poéticas, sino que lo hizo, indudablemente, por propia necesidad creativa, para dar una respuesta personal al estímulo proporcionado por el entorno natural. No cabe duda, pues, de que Dorothy Wordsworth fue otro miembro de la proverbial nómina de grandes mujeres que se esconden detrás de los grandes hombres de la historia. 
Tomar los diarios escritos en Alfoxden y Grasmere como una mera fuente secundaria para aproximarnos al estudio de la poesía de los dos autores canonizados nos ciega, como sugiere Frances Wilson (2008: 5), a la originalidad de la voz de Dorothy y a lo peculiar de las historias que en ellos nos relata. Por el contrario, el leer sus páginas por su valor intrínseco nos otorga la enorme recompensa de abrirnos los ojos ante una extraordinaria fuerza descriptiva que se manifiesta en toda serie de detalles sobre las naturaleza humana y no-humana que rodeaban a Dorothy; en particular, la de su amado Lake District. Ningún análisis, por detallado que este sea, puede hacer justicia a la belleza contenida en las páginas de sus diarios. Las anotaciones sobre los cambios de las estaciones, sobre la vida que se desarrollaba a su alrededor, sobre la suya propia, y sus cambio de ánimo nos muestran a una mujer tremendamente observadora, poseedora de un talento que, si dejamos de mirar su obra como mero accesorio a la de su hermano, nos permite verla brillar con luz propia.

Teniendo en cuenta los condicionamientos sociales de la época en la que vivió, Dorothy disfrutó de un grado de libertad inimaginable para muchas de sus contemporáneas de clase media, incluso si observamos en los diarios su papel fundamental en la esfera doméstica. Según nos cuenta Virginia Woolf (1986: 165-166), la vida que Dorothy y William escogieron les permitió en un primer momento vivir en el corazón de la naturaleza, dedicando cada día a tratar de desentrañar su significado y a escribir poesía. En aquellos primeros momentos de convivencia, con estabilidad económica y sin grandes obligaciones familiares, Dorothy podía dar largos paseos solitarios o pasar tranquilamente la noche entera hablando con Coleridge sin miedo a que su conducta fuese tachada de indecorosa. Asumió, sin embargo, una posición subalterna, al poner su vida y su trabajo al servicio de un bien que ella consideraba superior: la poesía de su hermano William, tanto si se trataba de transcribir sus poemas como de escribir un diario que él luego pudiese utilizar.

Dorothy empezó a escribir el diario de Alfoxden en el preciso instante en que supo que tendrían que abandonar la casa en la que habían vivido en los últimos seis meses. Deseosa de guardar de alguna forma lo que sabía que pronto iba a perder, empezó por describir la magnífica vista que la casa le ofrecía, del mismo modo que hoy nos lanzaríamos a hacer fotografías de nuestros espacios favoritos antes de abandonar un lugar querido. De hecho, muchas de las entradas de este primer diario tienen esa calidad de instantáneas, como si hubiesen sido escritas con prisa, con la intención de capturar - más que elaborar - cualquier objeto o vista al que estimase necesario dotar de presencia textual. Otras, sin embargo, nos proporcionan una estupenda muestra de lo que Thomas De Quincey describió como "her power of catching and expressing all the hidden beauties of natural scenery, with a felicity of diction, a truth and strength, that far transcend Gilpin, or professional writers on those subjects" (2009: 241). La primera entrada en el diario data del 20 de enero de 1798 y merece ser reproducida en su totalidad pues constituye uno de los mejores ejemplos de esa virtud que De Quincey alaba.

The green paths down the hill-sides are channels for streams. The young wheat is streaked by their lines of water running between the ridges, the sheep are gathered together on the slopes. After the wet dark days, the country seems more populous. It peoples itself in the sunbeams. The garden, mimic of spring, is gay with flowers. The purple-starred hepatica spreads itself in the sun, and the clustering snow-drops put forth their white heads, at first upright, ribbed 
with green, and like a rosebud when completely opened, hanging their heads downwards, but slowly lengthening their slender stems. The slanting woods of an unvarying brown, showing the light through the thin net-work of their upper boughs. Upon the highest ride of that round hill covered with planted oaks, the shafts of the trees show in the light like the columns of a ruin. (Wordsworth, 2001: 141)

Dorothy se quejaba con frecuencia de que no se le daba bien el dibujo; carecía, pues, de uno de los preciados accomplishments de las mujeres de clase media de su época. No le hacía falta, sin embargo, pues le bastaban las palabras. En sus manos, la hoja en blanco se convierte en un lienzo en el que capta de manera exquisita la vista que probablemente admira sentada frente a una ventana, trayéndonos desde las colinas más cercanas hasta la proximidad de su jardín, donde se concentra en el color, la forma y el movimiento de las flores, para luego llevarnos, como la ola que se aleja de la orilla, hacia la lejanía de los bosques. El alma que habita en cada ser vivo se nos sugiere sin pretensión alguna, con un estilo que confirma lo que Nabholtz admirase como "her 'picture-making power"” (1964: 118).

Si bien resulta evidente que los diarios de Dorothy se inscriben dentro de la tradición de lo pintoresco, es también notable su afán por encontrar una voz propia; incluso, en ciertos momentos, la articulación de una estética independiente (Newlyn 2007: 328). No hay rastro de Dorothy en esta primera entrada del diario; las frases están perfectamente equilibradas, escritas en un tiempo presente que le otorgan, siguiendo a Newlyn (2007: 328) "a celebratory mood - almost psalmic", al tiempo que el ritmo se acelera, como en respuesta al despertar del campo.

Por el contario, el comienzo de las segunda y tercera entradas, del 21 y 22 de enero, respectivamente, nos presentan a Dorothy sumergida activamente en el paisaje que está describiendo: "Walked on the hill-tops - a warm day"; "Walked through the wood to Holford" (Wordsworth, 2001: 141). En estas dos pequeñas anotaciones sobre las actividades realizadas en estos dos días, encontramos tanto el paisaje percibido como el sujeto perceptor: una Dorothy sentada bajo los abetos que nos hace partícipes de sus interrogantes: "Are the male and female flowers on separate trees?" (Wordsworth, 2001: 141). Resulta difícil no preguntarse sobre la posibilidad de que Dorothy extrapolase estas consideraciones a la separación de los géneros dentro de la especie humana, pero los diarios no dan cuenta alguna de ello.

Con relativa frecuencia da la impresión de que Dorothy no hubiera tenido tiempo para actualizar su diario, de manera que escribe de memoria en una sola sentada los contenidos de varios días. En algunas ocasiones, incluso, las prisas la llevan a resumir un día entero en una sola línea: Así, el 28 de marzo "Hung out the linen", el 29 "Coleridge dines with us", el 30 "Walked I know not where", mientras que el 31, simplemente, "Walked" (Wordsworth 2001: 150). Es este precisamente el verbo que Dorothy escoge con mayor frecuencia para dar comienzo a las anotaciones de su diario de Alfoxden. En abril de ese mismo año, posiblemente entusiasmada por la llegada de la primavera, una serie de entradas prácticamente idénticas dan cuenta de sus caminatas diarias por el bosque. El verbo walk conjugado en pasado da comienzo a doce de las veintisiete entradas para ese mes, pero la actividad de caminar se incluye en cada uno de esos días, a excepción de uno en el que Dorothy parece ser incapaz de recordar lo que hizo a juzgar por los puntos suspensivos que siguen a la 
introducción de la fecha del 19 de abril. Independientemente de que recogiera su actividad como "walked", "strolled", went" o "set forward to", sola, con William, con Coleridge o con ambos; de que lo hiciese en pleno día o a la luz de la luna, el diario de Alfoxden nos revela a una Dorothy en constante movimiento, siendo el caminar una de las actividades con las que más disfrutaba. La otra era la escritura.

Dorothy Wordsworth escribía constantemente, no sólo diarios, sino también cartas, poemas, pequeños relatos o cuadernos de viaje. El diario, sin embargo, resulta ser su medio favorito de expresión. Todo encuentra cabida en sus páginas, de lo sublime a lo mundano, el canto de los pájaros en perfecta convivencia con problemas intestinales. Dorothy siembra guisantes, arranca hierbajos, expresa su deseo de tener un libro de botánica, plancha, lee, pasea en el jardín, tinta y almidona la ropa, copia los poemas de su hermano, nos cuenta la vida de sus vecinos, sufre dolores de cabeza, está triste o alegre, camina y escribe incesantemente. En manos de Dorothy, el diario como forma de expresión se convierte en una poderosa narrativa del transcurso de la vida emocional, doméstica y artística de un grupo de seres extraordinarios (Levin, 2009: xi). El brevísimo registro que guardó en Alfoxden nos deja a Dorothy a punto de irse a dormir en Cross, después de haber caminado a Chedder el 17 de mayo de 1798. Año y medio después, empezaría a escribir en su casa de Grasmere el que se convirtió en su diario más conocido. Sus páginas nos ofrecen algunos de los mejores ejemplos de escritura de la naturaleza de la literatura inglesa.

El diario de Grasmere comienza con una nota melancólica. La primera entrada, de tono muy distinto al que nos encontrábamos al comienzo del escrito en Alfoxden, anticipa el ánimo cambiante de la autora que encontraremos con bastante frecuencia en sus páginas. En esta ocasión, la autora se niega a ausentarse del paisaje que describe; antes al contrario, su tristeza lo impregna todo. Si, tal y como asegura Wilson (2008: 128), el recurso literario favorito de Dorothy es la falacia patética, el lago le parece ahora aburrido y melancólico, en consonancia con una tristeza que sólo consigue aliviar ligeramente tras haber derramado un torrente de lágrimas (Wordsworth, 2001: 1). Sus hermanos William y John acababan de marcharse a Gallow Hill, cerca de Scarborough, para visitar a Mary Hutchinson, y Dorothy se quedaba sola en casa por primera vez desde que William y ella empezaran a compartir residencia en Dove Cottage.

Si el diario de Alfoxden estaba marcado por la pérdida del hogar, el de Grasmere celebra la llegada y asentamiento en uno nuevo (Newlyn, 2007: 331). Es un diario mucho más elaborado, escrito durante un período de tiempo más largo e intenso, que supuso importantes cambios para la vida de los Wordsworth. Nos deja constancia de la misma actividad frenética de Alfoxden, pero nos proporciona muchos más ejemplos - y muy interesantes - de esa excesiva sensibilidad orgánica de la que De Quincey daba cuenta; una sensibilidad que había hecho a la niña Dorothy temer tocar una mariposa por miedo a que no pudiera volar si alteraba el polvo de sus alas, o la había llevado a alimentar petirrojos hasta hacerlos entrar en su habitación, afición esta última que siguió cultivando hasta una edad bien avanzada (Gittings \& Manton, 1985: 26). Buena cuenta de esta sensibilidad nos la proporciona el siguiente extracto, en el que explica los motivos que la llevaron a volver a plantar una flor de fresa que de manera impulsiva había arrancado de una roca hacía sólo unos segundos: "[T]he little slender flower had more courage than the green leaves, for they were but half expanded and half grown, but the blossom was spread full out. I uprooted it rashly, \& felt 
as if I had been committing an outrage, so I planted it again - it will have but a stormy life of it, but let it live, if it can" (Wordsworth, 2001: 61).

No obstante, esa misma sensibilidad que la hacía tan solícita a las necesidades de los demás, también la hacía víctima - como no podía ser de otra forma - de un constante conflicto interno (Wilson, 2008: 18). Son muy frecuentes las referencias a dolores de cabeza, ataques de ansiedad o de melancolía y a cambios de humor que podían llevar a Dorothy de la carcajada al llanto en cuestión de segundos. Cinco días después de haber despedido a sus hermanos, la tranquilidad y reclusión del valle la vuelven a sumergir en una honda tristeza (Wordsworth, 2001: 4). En ocasiones, ni siquiera la vista más hermosa puede sacarla de ese estado de melancolía; de hecho, con frecuencia la hunden en ella. Así, tras describir un entorno casi idílico, "[e]verything green $\&$ overflowing with life $\&$ the streams making a perpetual song with the thrushes \& all little birds, not forgetting the stone chats", Dorothy se incluye de lleno en la escena con un breve "I grew so sad" (Wordsworth, 2001: 4). De manera parecida, después de una visita a Coleridge, "[t]he sunshine - the Green fields and the fair sky made me even sadder; even the little happy sporting lamps seemed but sorrowful to me" (Wordsworth, 2001: 89)

Son las viñetas de tranquilidad doméstica las que nos presentan a una Dorothy mucho más satisfecha. En la entrada del 6 de mayo de 1802, por ejemplo, el uso del presente continuo y las constantes referencias deícticas, así como lo errático de los signos de puntuación dotan al siguiente extracto de un estilo íntimo, conversacional que nos permite creer que estamos compartiendo tiempo y espacio con los protagonistas de la escena:

A sweet morning we have put the finishing stroke to our Bower \& here we are sitting in the orchard. It is one o clock. We are sitting upon a seat under the wall which I found my Brother Building up when I came to him with his apple [...]. It is a nice cool shady spot. The small Birds are singing - Lambs bleating, Cuckow calling - the Thrush sings by Fits, Thomas Ashburner's axe is going quietly (without passion) in the orchard - Hens are cackling, Flies humming, the women talking together at their doors - Plumb \& pear trees are in Blossom, apple trees greenish - the opposite woods green, the crows are cawing. We have heard Ravens. The Ash Trees are in blossom, Birds flying all about us. The stitchwort is coming out, there is one budding Lychnis. The primroses are passing their prime. Celandine violets \& wood sorrel for ever more - little geranium \& pansies on the wall (Wordsworth, 2001: 96).

A través de los canales visual y auditivo, la entrada consigue trasladar a quien la lee a la comodidad íntima del entorno doméstico en la interacción armoniosa de las naturalezas humana y no-humana.

Wilson observa que, en el último de los cuadernos que componen el diario escrito en Grasmere, Dorothy dedica prácticamente toda su atención a observar el paso de la primavera al verano y su efecto en el crecimiento de las plantas y la migración de las aves, y establece una distinción interesante entre la forma en la que Dorothy responde a la naturaleza ordenada del jardín, donde todo sucede "without a passion", frente a la belleza sublime de las colinas, los valles y los lagos más allá del entorno doméstico. En esas ocasiones en las que la belleza del valle es sobrecogedora, la autora no quiere describir una escena, sino expresar una idea: "in the excessiveness of the green she is saying something about the power and terror of what lies within" (Wilson, 2008: 168-169). Para Dorothy, pues, la 
seguridad emocional resultaría incompatible con cualquier tipo de exceso, incluso si este lo es de belleza.

Hay, sin embargo, otros sentimientos que encuentran expresión en el diario de Grasmere aparte de esa sensación de seguridad que le proporciona el entorno inofensivo de su jardín. Si la naturaleza al otro lado de la verja le supone una fuente de temor, también lo es de inspiración para muchas de las descripciones más sublimes del diario. Así, el 27 de julio de 1800 - que Dorothy escribe erróneamente como el 26 - el cambio en el color del lago en Rydale, otrora de tonos marrones por el efecto del sol, queda recogido como sigue:

The lake was now most still \& reflected the beautiful yellow \& blue \& purple \& grey colours of the sky. We heard a strange sound in the Bainriggs wood as we were floating on the water it seemed in the wood, but it must have been above it, for presently we saw a raven very high above us - it called \& the Dome of the sky seemed to echoe [sic] the sound - it called again \& again as it flew onwards, \& the mountains gave back the sound, seeming as if from their center a musical bell-like answering to the birds hoarse voice. We heard both the call of the bird \& the echo after we could see him no longer (Wordsworth, 2001: 14).

El graznido del cuervo reverberando en el fondo de la escena lleva a Dorothy a apreciar una suerte de conversación entre el pájaro y las montañas que los seres humanos en la escena sólo pueden presenciar extasiados. El extracto nos permite asimismo atisbar en la llamada del pájaro desapareciendo en la distancia un anticipo del himno del ruiseñor de Keats debilitándose "Past the near meadows, over the still stream,/ Up the hill-side" (1994: 233).

Dorothy deja constancia en sus diarios de un profundo amor por el entorno natural y una extraordinaria capacidad de respeto por su alteridad, independientemente de cómo pudiera afectar a su estado de ánimo. Este respeto se torna en veneración casi religiosa en algunas entradas, rasgo este eminentemente Romántico que se presenta en el estilo sencillo y falto de afectación tan característico de Dorothy, cuando el 3 de septiembre de 1800 nos describe la magnífica escena que se muestra ante sus ojos tras asistir a un funeral:

When we got out of the dark house the sun was shining \& the prospect looked so divinely beautiful as I never saw it. It seemed more sacred than I had ever seen it, \& yet more allied to human life. The green fields, neighbours of the churchyard, were green as possible $\&$ with the brightness of the sunshine looked quite Gay. I thought she was going to a quiet spot \& I could not help weeping very much (Wordsworth, 2001: 20; énfasis añadido).

Esta relación armónica entre la naturaleza humana y la no-humana de la que anteriormente había disfrutado en el jardín, se torna aquí en presencia sagrada; la naturaleza no-humana parece una divinidad a la que no hay que temer pues se percibe aliada de la vida humana. Cuales fueran sus sentimientos religiosos, estos parecían estimularse en presencia de una naturaleza, frente a la cual, Gittings y Manton nos aseguran, "belief came naturally as breath" (1985: 121).

Caminar no era para Dorothy solamente una forma de encontrarse con esta naturaleza no-humana, o de compartir un rato de actividad con un ser querido; era también un modo de encontrar su centro existencial siempre que caminaba sola. El diario de Alfoxden recoge largos paseos con Coleridge; en Grasmere su acompañante más habitual era William y, si 
bien no cabe la menor duda de que Dorothy disfrutaba de la compañía de su hermano, su experiencia del entorno natural se percibe distinta en soledad. De hecho, muchas de las descripciones que siguen esos paseos solitarios adquieren un tono menos descriptivo y más confesional. Si observamos la forma en la que la autora se incluye en los cuadros que pinta con sus palabras, no podemos evitar preguntarnos si se percibía como una de las criaturas frágiles que tan dada era a proteger o más bien como la valiente flor de fresa que volvió a plantar para permitirle vivir incluso desafiando a los elementos.

Para seguir indagando en esta pregunta, volvamos al tono melancólico que detectamos en el momento que inauguraba el diario de Grasmere. Si Frances Wilson tiene razón en asumir que Dorothy no acompañó a William en su visita a Mary Hutchinson dado que el motivo de la misma era proponerle matrimonio a la amiga de ambos, es bastante probable que Dorothy temiera el cambio que el nuevo estado de su hermano traería a su hogar. Sin nadie a su lado que pudiera distraerla del paisaje, da rienda suelta a sus sentimientos más escondidos, algunos de los cuales no habría admitido en otra ocasión. Hagamos ahora una lectura atenta de la entrada que escribió el 23 de junio de 1800 y comparémosla con la escrita el 15 de abril de 1802. En la primera, tras un largo paseo, Dorothy trata de aliviar su dolor de cabeza tumbada junto a un lago, mientras William pesca: "I lay under the wind my head pillowed upon a mossy rock \& slept about 10 minutes which relieved my headach" (Wordsworth, 2001:13; énfasis añadido). En la correspondiente a abril de 1802, Dorothy admiraba los mismos narcisos que su hermano luego inmortalizaría en el famoso poema; los de Dorothy "grew among the mossy stones about \& about them, some rested their heads upon these stones as on a pillow for weariness \& the rest tossed \& reeled \& danced \& seemed as if they verily laughed with the wind that blew upon them over the Lake, they looked so gay ever glancing ever changing" (Wordsworth, 2001: 85; énfasis añadido). Casi dos años separan las dos entradas del mismo diario, pero resulta curioso que Dorothy presente algunas de esas flores exactamente en la misma posición que ella había adoptado mientras trataba de librarse de ese dolor de cabeza; tanto las flores como Dorothy apoyan su cabeza en piedras musgosas. La deuda de William con la descripción de Dorothy en "I wandered lonely as a cloud" es evidente, si bien él escogió incluir sólo uno de los dos grupos de narcisos que ella describe en el diario: los que él presentó "fluttering and dancing in the breeze" (Wordsworth, 1986: 193), al tiempo que obvió aquellos que, como su hermana, utilizaron las piedras como almohada. Dorothy percibe al pequeño grupo de narcisos como ella se había sentido dos años atrás, cansada, incapaz de reír y bailar como el grupo más amplio; ¿es posible que se sintiera desplazada?

Wilson ha explorado en profundidad cómo la decisión de William de casarse afectó negativamente al ánimo de Dorothy. Es este un asunto en el que prácticamente todas sus biografías coinciden y que resulta fácil corroborar tras echar un vistazo a las páginas del diario escritas en los meses previos a la boda. William se casaría con Mary en octubre de 1802 y había planeado viajar con Dorothy a Francia antes de la boda, en el verano, para visitar a Annette Vallon y a la hija de ambos. La tensión que debió experimentar en aquella época por el pesar que le causaba pensar en la posición en la que Annette y Anne-Caroline quedarían relegadas tras la boda resultó ser casi insoportable a juzgar por las referencias al insomnio, los constantes dolores de cabeza y un permanente decaimiento anímico que la lleva incluso a descuidar su querido jardín, "overrun with weeds" (Wordsworth, 2001: 117). 
Incluso su percepción del canto de los pájaros se ve alterada en la entrada del sábado 19 de junio, cuando describe "our own Thrush shouting with an impatient shout - so it sounded to me. The morning was still, the twittering of the little Birds was very gloomy" (Wordsworth, 2001: 112; énfasis añadido). Su reacción ante la caída del nido que unas golondrinas habían estado construyendo durante diez días da muestra del estado de extrema sensibilidad en el que se encontraba, pues el accidente parece hacerse eco de sus temores frente a los cambios que se cernían en el horizonte. "Poor Little creatures", escribe, "they could not themselves be more distressed than I was" (Wordsworth, 2001: 115). Por fortuna, las golondrinas consiguieron terminar su nido el 6 de Julio, justo a tiempo de proporcionar una nota alegre que llevarse de viaje a Francia dos días después. Dorothy podría haber tomado perfectamente la reconstrucción del nido como un buen presagio de la continuidad de su vida feliz con su hermano, incluso con Mary como nuevo miembro de la familia.

No obstante, Dorothy Wordsworth era consciente de que la vida no podía continuar igual; de que ella misma cambiaría. La certeza de que, en palabras de Wilson (2008: 32) "attachment for Dorothy was a continuation of selfhood", hace su despedida temporal de Dove Cottage mucho más conmovedora ya que, al decir adiós a cada objeto y criatura, se está despidiendo de una parte de ella misma que nunca volverá una vez que Mary se transforme en la esposa de su hermano: "I must prepare to go - The Swallows I must leave them the well the garden the Roses all - Dear creatures! They sang last night after I was in bed - seemed to be singing to one another, just before they settled to rest for the night. Well I must go - Farewell. - - -" (Wordsworth, 2001: 119).

Si bien la vida en Dove Cottage ciertamente cambió, no lo hizo de la manera que Dorothy temía. El núcleo familiar se amplió para incluir a Mary y a los hijos que fueron naciendo y Dorothy fue siempre un miembro central en el enclave familiar. Juntos compartieron una vida de momentos felices y trágicos, con Dorothy disfrutando del nacimiento de sus sobrinos y sobrinas y sufriendo la muerte de algunos de ellos. El cambio más significativo fue el que afectó a su escritura, pues el diario de Grasmere termina de manera abrupta tres meses después de la boda. Dorothy siguió escribiendo cartas y poemas; sin embargo, estos carecen del grado de intimismo y espontaneidad de los diarios, al no tener como destinatarios el de estos: su hermano William. Es posible que la autora pensase que una vez que Mary ocupara su lugar como la confidente más directa de William, poco sentido podía tener el seguir escribiendo un diario del que nadie podría sacar provecho. En esa creencia, de nuevo, se dejó engañar por su humildad. Los diarios de Alfoxden y de Grasmere nos revelan a Dorothy Wordsworth como una de las más exquisitas escritoras de la naturaleza, y son una fuente de placer para otros muchos lectores y lectoras aparte de su querido hermano. Tras su lectura, resulta imposible volver a visitar la poesía de William sin detectar la huella de Dorothy, al tiempo que se atisba una identificación con algunos aspectos de la naturaleza no-humana que trasciende la relación experimentada por su hermano y que, con toda probabilidad, evidenciaría un estudio comparativo más exhaustivo. Si bien resulta reduccionista considerar una base exclusivamente biológica en esta peculiar relación, podemos afirmar - especialmente si atendemos al retrato que el propio Wordsworth esboza de su hermana en poemas como "To a Butterfly" o "The Sparrow's Nest" - que la de Dorothy fue, a todas luces, la posición epistemológica privilegiada. De haber sido otras sus circunstancias, de haber tenido la oportunidad de dedicarse de manera casi exclusiva a 
la poesía, no cabe duda de que su producción habría sido mayor y habría recibido mucho antes la atención que su obra merece, apreciada por su valor intrínseco y no como un mero complemento a la poesía de su hermano.

\section{REFERENCIAS BIBLIOGRÁFICAS}

Carretero González, M. 2010. "Ecofeminismo y análisis literario.” Ecocríticas. Literatura y medio ambiente. Eds. C. Flys Junquera, J.M. Marrero Henríquez y J. Barella Vigal. Madrid y Frankfurt am Main: Iberoamericana / Vervuert. 177-189.

Daly, M. 1978. Gyn/Ecology: The Meta-Ethics of Radical Feminism. Boston: Beacon Press.

De Quincey, T. 2009. "From Recollections of the Lake Poets." Dorothy Wordsworth. Ed. S.M. Levin. Nueva York \& Londres: Pearson Education \& Longman. 237-242.

GitTings, R. \& Manton, J. 1985. Dorothy Wordsworth. Oxford: Clarendon Press.

Griffin, S. 1978. Woman and Nature: The Roaring Inside Her. Nueva York: Harper and Row.

KeAts, J. 1994. “Ode to a Nightingale.” The Complete Works of John Keats. Ware: Wordsworth Editions Ltd.

Levin, S.M. (ed.). 2009. Dorothy Wordsworth. Nueva York \& Londres: Pearson Education \& Longman.

Mellor, M. 1997. Feminism \& Ecology. Cambridge: Polity Press.

Nabholtz, J.R. 1964. "Dorothy Wordsworth and the Picturesque". Studies in Romanticism 3, 2: 118-128.

Newlyn, L. 2007. "Dorothy Wordsworth's Experimental Style". Essays in Criticism 57, 4: 325-349.

Puleo, A. 2000. Filosofía, género y pensamiento crítico. Valladolid: Secretariado de Publicaciones e Intercambio Editorial de la Universidad de Valladolid.

Starhawk. 1979. The Spiral Dance: A Rebirth of the Ancient Religion of the Great Goddess. Nueva York: HarperCollins.

WiLson, F. 2008. The Ballad of Dorothy Wordsworth. Londres: Faber \& Faber.

Woolf, V. 1986. The Second Common Reader. San Diego, Nueva York \& Londres: A Harvest Book, Harcourt, Inc.

Wordsworth, D. 2001. The Grasmere and Alfoxden Journals. Ed. P. Woof. Oxford: Oxford University Press.

WORDSWORTH, D. \& W. 1986. Home at Grasmere. Extracts from the Journal of Dorothy Wordsworth and from the Poems of William Wordsworth. Ed. C. Clark. Harmondsworth: Penguin Books.

WORDSWORTH, W. 1994. The Works of William Wordsworth. Ware: Wordsworth Editions Ltd. 